\title{
Pursuit for Simple Power Conditioner and System Construction of Photovoltaic Power Generation as Veranda Solar
}

\author{
Keiju Matsui ${ }^{\text {a, }}$, Eiji Oishi $^{\text {a }}$, Mikio Yasubayashi ${ }^{\text {b }}$, Masayoshi Umeno ${ }^{\text {b }}$ \\ Hideo Uchida ${ }^{\mathrm{b}}$, Yasutaka Kawata ${ }^{\mathrm{b}}$, Masaru Hasegawa ${ }^{\mathrm{b}}$ \\ ${ }^{a}$ Minna-denryoku, Inc., Setagaya Monozukuri Gakko, 210, Setagaya154-0001, Japan \\ ${ }^{\mathrm{b}}$ Chubu University, Kasugai 487-8501, Japan \\ *Corresponding Author: keiju@ @isc.chubu.ac.jp
}

\begin{abstract}
Utility-interactive photovoltaic power generations have been accepted and spread widely. Various innovative power conditioning systems have been also studied. In domestic utilization, the actual application of such solar panels is almost installed on top of the roof of the detached house. However, some residents living in the apartment house are having a fairly strong desire to contribute for energy saving due to natural energy generation. The generating power in such case is fairly reduced, so the system construction should be balanced with the reduced power. Thus, it is necessary to improve the construction for simple one. In this paper, in order to give a reply, simple and concise photovoltaic power generating systems are examined. Considering fairly reduced generation power and narrow space of installation in the apartment houses, the system constructions should be compact. The circuit which gratifies their wishes are presented and discussed. These solar panels can be easily connected like usual home appliances having attached plug for connection. For reversed power flows and increasing harmonics, their protecting circuits are installed in the input power line. Such systems will be presented and discussed.
\end{abstract}

Keywords: PV panel1, harmonic elimination2, forward converter3, PV power generation4, power interruption 5

\section{Introduction}

Preserving the developments of economy, science and technology with conquering the problems against environmental conservation is an important assignment for humanity of the world. The usage of the renewable energies is expected to be able to mitigate such problem, which is an important means ${ }^{(1,2)}$. Among them, photovoltaic power generation have various advantages such as usage of inexhaustible and unpolluted sunlight, which is advantageous for maintenances, easily construction from small power to large one, and can be installed in various location including city center. In such a way, this power generation system is a promising one with bright future.

As a utilization form, utility interactive power generation system has been accepted and widely spread. In such power generation system, it is necessary to install the chopper, where the lower voltage of solar cell should be boosted to suitable voltage and inverter where the dc power of boosted voltage should be converted to ac power in the power system ${ }^{(3,4)}$.

The power conditioners-PCS including inverter have been presented in various systems so far ${ }^{(5-8)}$. However, it is necessary to reduce the cost even more. It is said that the system is approaching to an ideal ones with respect to efficiency and the like, but that cost would prevent wide spread if there is no public financial support.

In such discussions, there are many subjects to be solved to utilize the PV power in utility interactive power generation. Even more, various safeguard equipment required according to regulations make the cost increase Thus, it is required to obtain even lower cost PCS and the like. In an extremely lower capacity PCS as discussed in this paper, a way of handling would be different compared to conventional ones. In such case of reduced generating power, quantities of reversed power to the power system would be small, where another mitigated regulation or deregulation would be approved. Thus, in such photovoltaic power generation systems, there are so many subjects to be resolved ${ }^{(9)}$.

Under such circumstances accepted by electrical utility 
industry as recognitions and assessments for renewable energy, spread of application is strongly sustained by financial supports of public organizations, and a lot of consumers are hoping to install such PV power generation system. For ordinary homes, installation of solar panel is restricted to house having roofs. However, a lot of residents living in the apartment house also wish to install the PV panel under considering resource conservation and public financial support.. In the present situation, however, there is no scheme to perform these requirements ${ }^{(10)}$. In this paper some simple PV generation systems and the components for such residents are presented and discussed.

\section{Pursuit for Simpler Power Conditioner}

\subsection{Elimination of specific lower order harmonics}

In this chapter, in order to pursue simple wave for power conditioner, harmonic elimination methods for lower order harmonics with small power is presented and examined. Fig. 1 shows conventional single bridge circuit used in these situations. Because of simple way, a particular waveform calculated in advance is given at terminals $\mathrm{A}$ and $\mathrm{B}$ to eliminate specific lower order harmonics like by feed forward control which are fifth, seventh order harmonics in this case. It is more efficient because of lower order one.

The pole voltages relative to neutral point $\mathrm{O}$ is shown in Fig.2(a) and (b) as $\mathrm{V}_{\mathrm{A}}$ and $\mathrm{V}_{\mathrm{B}}$, respectively. In general, in utility- interactive operation, the sinusoidal current is a goal to inject to the utility with minimum harmonics. In order to achieve such purpose, by means of feedback control following the sinusoidal waveform of grid voltage, high frequency switching method is accepted. In this method, however, it is necessary to provide current detectors for making sinusoidal wave or hysteresis control circuit, so the total cost is increased and reliance would be somewhat lowered because of increased number of parts and sophisticated circuits and the like. Thus, it is more effective to improve the control schemes toward simple one. The objective of this chapter is to present a concise control scheme like feed forward control having simpler scheme, where single waveform having simple pattern resolved in advance is injected in the network.

The waveforms of just mentioned in Fig.2(a) and (b) are derived with such intentions of obtaining a simpler waveform where, for example, fifth and seventh order harmonics are eliminated. In order to eliminate such harmonics at the same time, following procedure is executed, so the solution can be obtained.

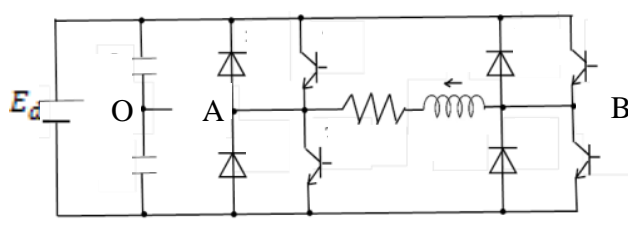

Fig.1. Full bridge inverter.

(a) $\mathrm{V}_{\mathrm{A}}$

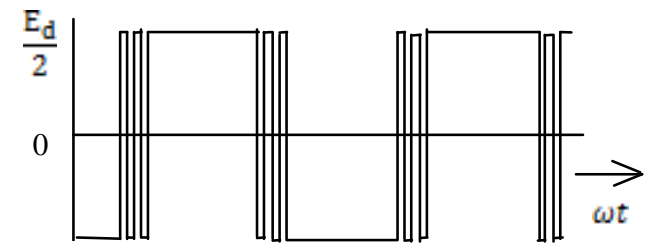

(b) $\mathrm{V}_{\mathrm{B}}$

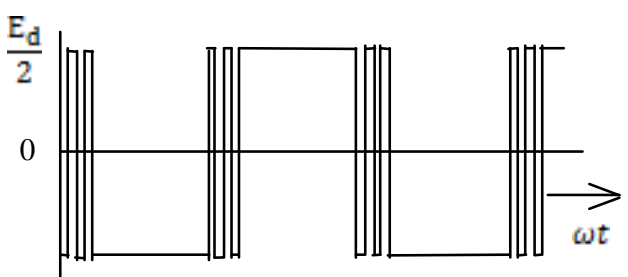

(c) $\mathrm{V}_{\mathrm{AB}}$

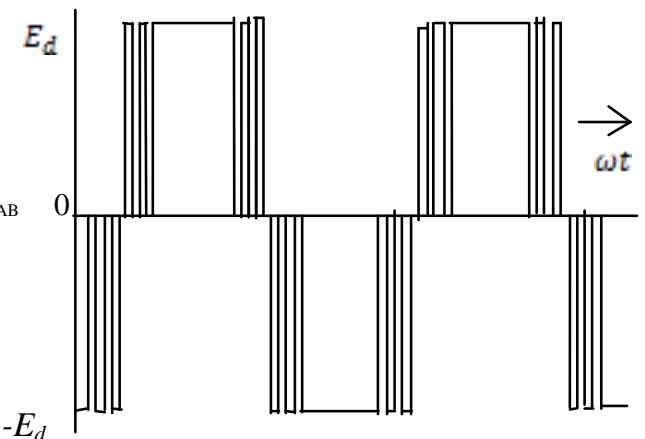

Fig.2. Waveforms; pole voltages (a) and (b); line voltage (c).

In the figure, waveform is switched the polarity two times in a quarter cycle, $\alpha_{1}$ and $\alpha_{2}$ which are phase angle from time origin, respectively. The waveforms are constructed in quarter symmetry. By varying angles, the corresponding zero harmonic can be obtained. Firstly, $\alpha_{1}$ and $\alpha_{2}$ are varied for fifth order harmonic to obtain the zero harmonic line for the first intension. For the other harmonic elimination, seventh order, analogous zero voltage line can be obtained by varying another $\alpha_{1}$ and $\alpha_{2}$ once again. As described later, the solution of simultaneous zero voltage values for fifth and seventh order harmonics could be obtained.

Fig.2(c) shows the line voltage waveform injected into the network. In such a way, the values of $\alpha_{1}$ and $\alpha_{2}$ for fifth and seventh orders are resolved as pole voltage. After that, the line voltage can be calculated by equation $V_{A B}=V_{A}-V_{B}$ where triplen harmonics can be eliminated by giving phase difference of $120^{\circ}$. That operation principle is well known 
in symmetrical three phase circuit theory. Also in this single phase for utility-interact, this theory can be applied favorably. From such reasons, the fifth, seventh and triplen harmonics can be entirely eliminated.

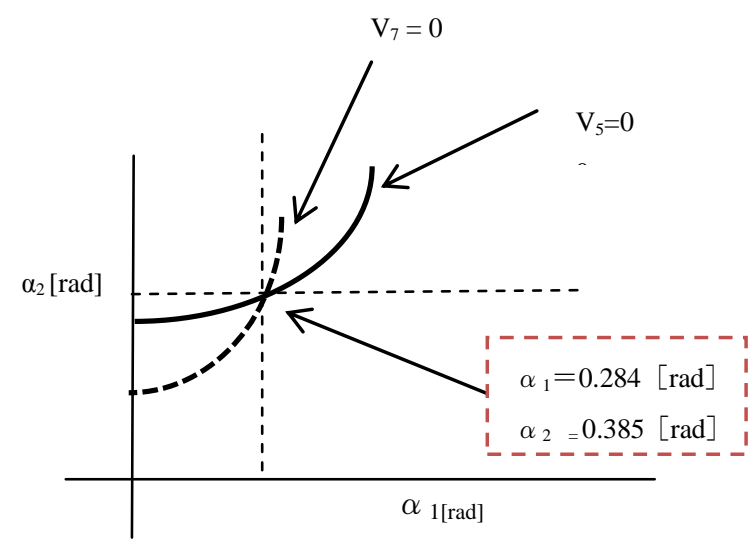

Fig.3. Solution diagram for fifth and seventh harmonics eliminated.

As just described explanation, actual solution can be obtained as follows; Fig. 3 shows an auxiliary figure to resolve the $\alpha_{1}$ and $\alpha_{2}$ where the fifth and seventh order harmonics are zero simultaneously at the intersection point.

The waveform in Fig.2(a) is expanded in Fourier series. The sub-expansions can be obtained as in (1) for $\mathrm{V}_{5}=0$ and in (2) for $\mathrm{V}_{7}=0$. In these equations, $\alpha_{1}$ and $\alpha_{2}$ are made varied to resolve for fifth harmonic. In the same way, $\alpha_{21}$ and $\alpha_{22}$ are varied for seventh harmonic, so that two zero line curves are obtained. The intersection point of two curves becomes $\mathrm{V}_{5}=\mathrm{V}_{7}=0$ that is the solution of switching angles.

$$
\begin{aligned}
& \alpha_{2}=\frac{1}{5} \cos ^{-1}\left(\cos 5 \alpha_{1}-\frac{1}{2}\right) \\
& \alpha_{22}=\frac{1}{7} \cos ^{-1}\left(\cos 7 \alpha_{21}-\frac{1}{2}\right)
\end{aligned}
$$

The switching angle in

Fig.2(a) and (b) could be obtained in such manner that is $\mathrm{V}_{5}=\mathrm{V}_{7}=0$ where $\alpha_{1}=0.284$ and $\alpha_{2}=0.385$. Fig. 4 shows harmonic spectrum for the waveforms in Fig.2. As can be seen, Fig.4(a) is for pole voltage which contains triplen harmonics that is third and its multiple orders. Fig.4(b) is the harmonic spectrum of waveform in Fig.2(c) which can be obtained from phase difference by $120^{\circ}$ between $V_{A}$ and $\mathrm{V}_{\mathrm{B}}$ as $\mathrm{V}_{\mathrm{AB}}=\mathrm{V}_{\mathrm{A}}-\mathrm{V}_{\mathrm{B}}$, where triplen harmonics are eliminated. In such manner, fifth and seventh plus triplen harmonics can be entirely eliminated.
$150 \mathrm{~V}$

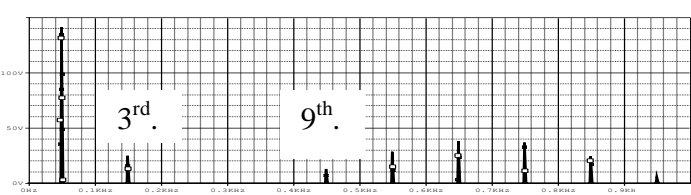

frequency

$1 \mathrm{kHz}$

(a)

$150 \mathrm{~V}$

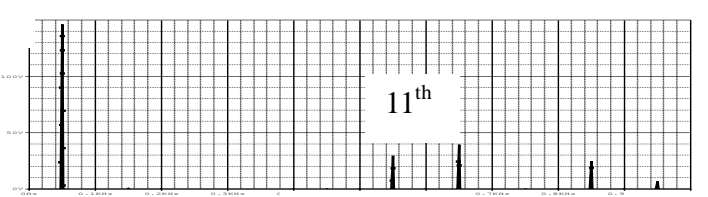

frequency

$1 \mathrm{kHz}$

(b)

Fig.4. Harmonic spectrums: pole voltage $\mathrm{V}_{\mathrm{A}}$ (a), line voltage $\mathrm{V}_{\mathrm{AB}}(\mathrm{b})$.

\subsection{Optimum PWM pattern of further improvement for harmonic characteristic}

In the above section, the harmonic elimination method is up to seventh order plus triplen harmonics, for example, $\mathrm{V}_{3}$ $=\mathrm{V}_{5}=\mathrm{V}_{7}=\mathrm{V}_{9}=0$, etc. The existing lower order is eleventh harmonic, which could be suppressed by filter inductor and the like. If this installed inductor is increased, it might be concerned about its large size and subsequent increased cost.

In this chapter, another solution to reduce that harmonic will be examined. In just mentioned chapter, the number of switching is twice for $\alpha_{1}$ and $\alpha_{2}$. Another method of more increasing the number of switching could be realized. In the fundamental policy of our study, however, system should be constructed in concise and that control should be simple such as with minimum number of switching. And yet, harmonic characteristic should be satisfactory. Firstly, the evaluation or performance index is introduced by means of weighting function method for harmonic characteristic. Thus,

$$
V_{h}^{2}=\left(V_{5} / 5\right)^{2}+\left(V_{7} / 7\right)^{2}+\left(V_{11} / 11\right)^{2}
$$

In this equation, the minimum value is solved by varying $\alpha_{1}$ and $\alpha_{2} . V_{h}$ is the effective value of harmonics for evaluation, where each term is divided by its order like $V_{5} / 5$.

By means of calculating the effective value using the weighting factor, the minimum value can be obtained as the optimum value. Fig.5 is a figure to obtain the optimum 
value from minimum effective value in (3). Firstly, $\alpha_{1}$ and $\alpha_{2}$ are varied to obtain the minimum value. In such a way, that minimum point is plotted according to varying $\alpha_{1}$, so the curve can be obtained as shown in the figure.

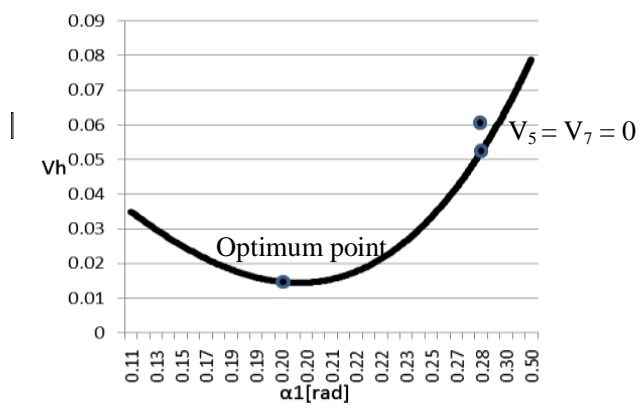

Fig.5. Resolving optimum value by varying $\alpha_{1}$ and $\alpha_{2}$.

The minimum of the effective value of the objective lower order harmonics represented in Fig.5 is $V_{h}=0.016$ as shown by a circle, where $\alpha_{1}=0.196 \mathrm{rad}$ and $\alpha_{2}=0.286 \mathrm{rad}$. In the previously mentioned results of , where $\alpha_{1}=0.196$ rad and $\alpha_{2}=0.286 \mathrm{rad}$, the minimum value is shown by $\mathrm{V}_{\mathrm{h}}$ $=0.058$ which is increased almost by three times. As can be seen, the proposed method is significantly dominant compared to the usual specific harmonic elimination.

Fig.6(a) shows the output voltage waveform at eliminating the fifth, seventh and triplen harmonics, that is $\mathrm{V}_{3}=\mathrm{V}_{5}=\mathrm{V}_{7}=\mathrm{V}_{9}=0$, etc. Fig.6(b) shows the harmonic spectrum of that waveform. As can be seen that the specific lower order harmonics are completely eliminated and do not appear. The lowest order is eleventh whose value is about $20.1 \%$ relative to fundamental one. Fig.7(a) is the minimization method of the lower order harmonics. The waveform pattern is analogous to just mentioned method. In the appearance of harmonic spectrum in Fig.7(b), however, as can be seen that the orders of up to eleventh are satisfactorily suppressed, though they do not made zero. The significant lowest order is $13^{\text {th }}$ whose value is fairly large of $7.6 \%$. Comparing both figures, the harmonic minimization method can be considerably superior.

Fig. 8 shows current waveforms of $120^{\circ}$ rectangular
$150 \mathrm{~V}$

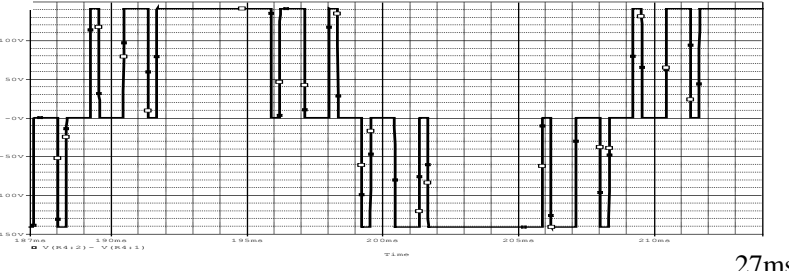

(a)

$150 \mathrm{~V}$

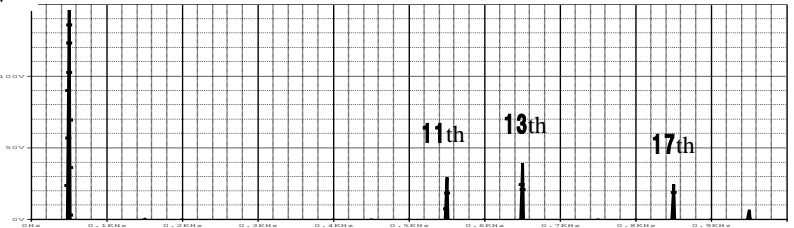

(b)

$1 \mathrm{kHz}$

Fig.6. $5^{\text {th }}, 7^{\text {th }}$ and triplen elimination method; waveform (a), harmonic spectrum (b).

$150 \mathrm{~V}$

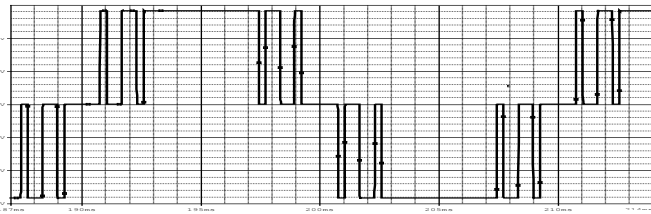

(a)

$27 \mathrm{~ms}$

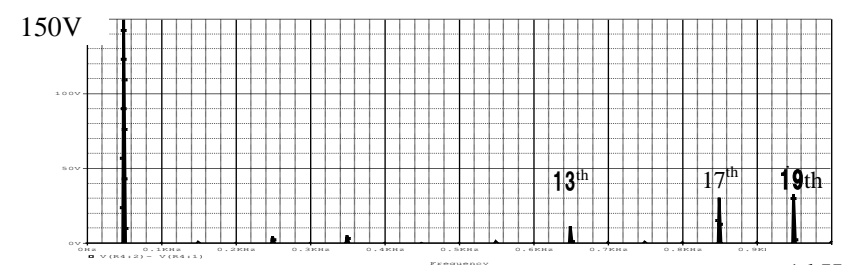

(b)

Fig.7. Minimization for lower order harmonics method; waveform (a), harmonic spectrum (b).

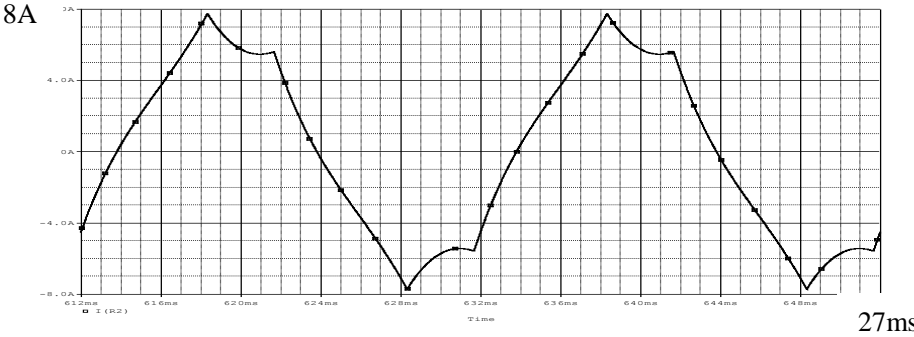

(a)

$8 \mathrm{~A}$

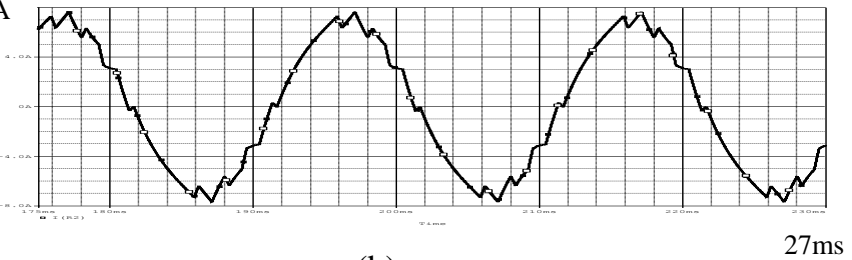

(b)

Fig.8. Current waveforms; $120^{\circ}$ rectangular (a), harmonic minimization (b). 
wave method (a) and harmonic minimization method (b). In the upper figure, the current ripple is much increased, in which the harmonics standard in JIS could not be cleared.

Table 1 represents comparison between specific fifth and seventh harmonic elimination method and minimization method of specific lower order harmonics. For the fifth and seventh elimination method, that is $\mathrm{V}_{5}=\mathrm{V}_{7}=0$, the lowest order harmonic becomes fairly large, $\mathrm{V}_{11}=0.201$. On the contrary, in the method of specific lower order harmonics, though fifth and seventh order harmonics are a little generated, such values are much reduced and relative value of $V_{11}$ is 0.008 . The total effect value of harmonic can be much suppressed from 0.065 to 0.050 that is satisfactory result. From these results, it can be confirmed that the minimization method for specific harmonics is effective solution.

Table 1. Comparison of output harmonics, relative to $\mathrm{V}_{1}$

\begin{tabular}{|c|c|l|l|c|c|c|}
\hline & $\alpha_{1}$ & $\alpha_{2}$ & $V_{h}$ & $V_{5}$ & $V_{7}$ & $V_{l I}$ \\
\hline Elim.of 5th, 7 th & 0.284 & 0.345 & 0.065 & 0 & 0 & 0.201 \\
\hline Minim. $5^{\text {th }}, 7^{\text {th }} 11$ th & 0.196 & 0.287 & 0.050 & 0.030 & 0.034 & 0.008 \\
\hline
\end{tabular}

\section{Actual Circuit Configuration of Indoors} Wiring

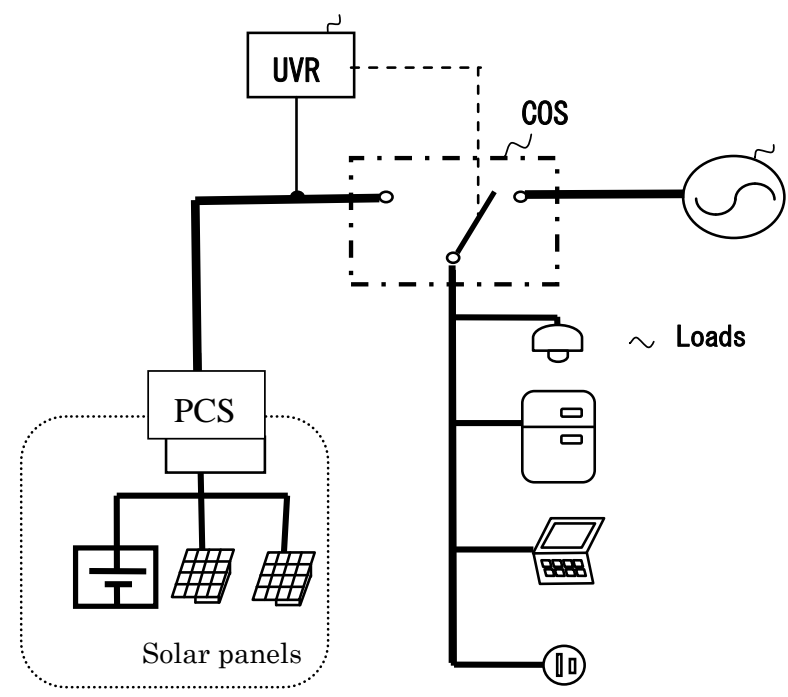

Fig.9. Actual circuit configuration of indoors wiring.

Fig.9 shows an example of indoors wiring from PV panel to utility network. As PV panel installed in veranda, there are various types such as from board type panels (BTP) to flexible PV panels (FSP) which can be rolled up like a curtain. In BTP, PCS in a thin box is attached to reverse side of board. These are selected in accordance to installed location. For usual roof installation type, a group of diodes protecting the reverse current, handling switches to each line and circuit breaker of lines with double polar and dual element are installed in the connection box. In this case, handling switch is operated by manual, while the circuit breaker is made turned off by over current. In thin type PCS, manual switches and line circuit breakers are installed in PCS. In FSP, PCS could be attached to outer side of cylindrical box.

Firstly, a certain exclusive line is provided where corresponding specific loads are connected. Consequently, the loads are connected to the PV panel. It is necessary to avoid the large power load to this line. This line is used for specific important load with reduced power at interruption. For emergency power supply at interruption which is positively used at night, it is necessary to install the battery as optional power supply. Because of minimum capacity of exclusive line at interruption, battery capacity can be also reduced. Recently, domestic battery storage systems are proposed and available. It might be considered to connect them this exclusive line. It is necessary to supply from the power system to charge, but it would be prospective subject. Thus, the line from PV panel uses the existing one. In order to utilize as the emergency power supply at disaster, it is important to select sufficiently and connect them as the important apparatuses at interruption.

For example, the important apparatuses at disaster such as light equipment, $\mathrm{PC}$ for collecting information, charger for cellular phone and refrigerator to protect foods are connected to this line. For the conventional refrigerator, however, the large rush current flows at turned-on, so this PV power could not correspond such power. Thus, the inverter type refrigerator can be used which suppresses such rush current and mitigates. Consequently, the inverter type would be recommended.

When the generating PV power is reduced and then the power supplying is stopped, the under voltage relay (UVR) detects this and the change-over switch (COS) switches from PV side to the network side immediately. As the power is supplied again to the specific loads with a short time delay, the supplying performance cannot be deteriorated except a minimum instantaneous interruption. After a negligible short interruption, the specific loads can be supplied satisfactorily.

When the PV power is increased, UVR functions and the COS switches to PV side. Thus, the specific loads can be supplied regardless of the night and day. In this example, the islanding operation is examined and discussed. As power capacity, however, connected load is 
restricted to relatively reduced loads. However, the installation work is very simple which brings lower cost. Under such situations, the authors expect and wish to spread such solar application.

\section{Conclusions}

It is a truth that many residents living in apartment house not only have interesting, but also desire to install the PV panel. An adequate power conditioner to be installed is discussed as an actual circuit. In such small generating power, reducing the power conditioners cost is more important compared to PV panel cost. The reason is; PV panel cost is reducing in proportion to reducing the number of panels, while power conditioner cost is not reducing in proportion to the generating power, because the number of power conditioner is single in general.

In such settlement at a narrow space like verandas, amounts of generating power is restricted, so lower cost is required even more. In such scheme, if the PV panel is handled as like home apparatuses having attachment plug, the settlement cost can be much reduced. If the unified specification is introduced and the mass production is promoted, even lower cost apparatus is supplied in the market. In such situation, the proposed simple power conditioner can bring with much more low cost favorably.

\section{Acknowledgment}

This research is mostly supported by a grant of the NEDO (New Energy and Industry Technology Development Organization). We would like to express our appreciation to who it may concern about this project.

\section{References}

(1) Yan $\mathrm{Hu}$, Keiju Matsui, Takashi Sugiyama, Kenji Ando and Isamu Yamamoto : "3kW Utility-interactive Power Conditioning System using Forward Converter", Proceedings of Static Power Converter Meeting in IEEJ, SPC-94-89, pp.49-56, 1994

(2) Sakae Shibasaki, Isao Takahashi, Shinzo Sakuma, Noriyasu Mimura, Yoshihiko Asano: "Small Quantity and Thin Type 200W Inverter for Photovoltaic Systems", Proceedings of National Convention in IEEJ,739, pp.4-50-51, 1997

(3) Edit: Ohmsha : "Guide Book of Technical Standard Concerning Electrical Equipment”, 1st edit., pp.52-63, pp.376-382, 2013

(4) Technical Committee for Customer Equipment, edit. : "Indoor Wiring Regulation - electrical code for customer equipment", Electric Association of Japan,pp.858-860, 2012

(5) R.L.Steigerwalt, A.Ferraro, and R.G.Turnbull : "Application of power transistors to residential and intermediate rating photovoltaic power conditioners', IEEE Trans. Ind Appl, IA-19, no.2, pp. 254-260, 1983

(6) A.Khoder, K.Al-Haddad and V.Rajagopalan : "Innovative utility-interactive $\mathrm{dc}$ to ac power conditioning system". Conference Record of 1985 IEEE IAS Annual Meeting, Toronto, pp. 1151-1155, 1985

(7) Chihiro Okado : "Development of inverter for photovoltaic generation", Proceedings of 10th Photovoltaic Generation System symposium, Tokyo, Japan, 1993, pp. 411

(8) R.L.Steigerwalt, B.K.Bose and P.M.Szczesny: "Design and construction of an advanced power conditioning subsystem for small photovoltaic applications", Sandia Report, SAND 83-7037, 1985

(9) Satoshi Naruse, Keiju Matsui, Shiro Hirose: "Utility-interactive forward converter with double switches", Proceedings of national convention in IEEJ, no.839, 1999

(10) Keiju Matsui, Eiji Oishi, Yasutaka Kawata, Mikio Yasubayashi, Masayoshi Umeno, Hideo Uchida, Masaru Hasegawa: "Simple and Concise Photovoltaic Power Generation Systems installed in Verandas of Apartment House", The IEEE APCCAS 2014, the 12th of the biennial Asia Pacific Conference on Circuits and Systems, B6P-D-05, pp.567-570, 2014 